\title{
HYPERTENSION
}

\section{NEW ROLES FOR}

CUL-3 IN THE KIDNEY

Mutations in cullin-3 (CUL-3) that cause familial hyperkalaemic hypertension $(\mathrm{FHHt})$ have previously been shown to abrogate degradation of WNK kinases, which regulate the renal $\mathrm{NaCl}$ cotransporter (NCC). Now, new findings from

David Ellison and colleagues demonstrate that CUL-3 403-459del is a gain-offunction mutant that exhibits increased activity compared with wild-type CUL-3.

WNK kinases have been a major focus of Ellison's laboratory for the past 14 years and this study was a continuation of their efforts to understand the association between WNK kinases and FHHt. First, they used a series of in vitro techniques to assess the function of CUL-3 403-459del.

"Our in vitro studies showed that the mutant CUL-3 dramatically enhances binding to, and ubiquitylation of, kelch-like protein 3 (KLHL3), which has a role in WNK kinase degradation," explains Ellison. "The resulting reduction in KLHL3 abundance leads to WNK kinase accumulation."

Next, the researchers used mice with nephron-specific deletion of Cul-3 to test the hypothesis that loss of Cul-3 function leads to $\mathrm{FHHt}$ via an increase in WNK kinase levels. "These Cul3\% mice generated some unexpected results that reveal new roles for CUL-3 in the kidney," says Ellison. "Although WNK and NCC were increased, they did not have an $\mathrm{FHHt}$ phenotype, but developed polyuria and salt wasting, which was associated with almost complete absence of activated $\mathrm{Na}-\mathrm{K}-\mathrm{Cl}$ cotransporter 2 and aquaporin 2 in the renal medulla." Based on these findings, the researchers suggest that CUL-3 may regulate pathways involved in fluid and electrolyte homeostasis in addition to NCC. Cul3 deletion also resulted in renal dysfunction, inflammation and fibrosis, indicating a new pathway for kidney injury.

Ellison and colleagues now aim to determine the mechanism by which loss of 57 amino acids in CUL-3 leads to a gain-of-function, and to characterize the regulation of aquaporin 2 and non-distal convoluted tubule ion transport pathways as well as the potential effects of CUL-3 knockout on the nuclear factor erythroid 2-related factor 2 /kelch-like $\mathrm{ECH}$-associated protein 1 pathway. They hope that manipulation of CUL-3 effects in the kidney will lead to new avenues for the prevention of chronic kidney disease.

Jessica K. Edwards

Original article McCormick, J. A. et al. Hyperkalemic hypertension-associated cullin3 promotes WNK signaling by degrading KLHL3. J. Clin. Invest. doi: 10.1172/JCI76126 